\title{
Contribuições da neurociência na compreensão do processamento sintático
}

\author{
Contributions of neuroscience to the understanding of syntactic processing
}

\author{
Rossana Kramer \\ Mariana Terra Teixeira \\ Pontifícia Universidade Católica do Rio Grande do Sul - PUCRS - Porto Alegre - Rio Grande do Sul - Brasil
}

\begin{abstract}
$\longrightarrow$
Resumo:Este trabalho procura contribuir para a compreensão da natureza da linguagem e do cérebro humano através da análise de estudos realizados em processamento sintático. Os estudos do processamento sintático da linguagem ainda constituem-se um desafio para a neurociência. No entanto, graças aos avanços tecnológicos, técnicas avançadas vêm sendo utilizadas para investigar como o cérebro armazena e processa informação, sendo capaz de construir uma sentença. Este trabalho abordará estudos que aplicaram tarefas de compreensão de estruturas sintáticas utilizando a Imagem de Ressonância Magnética Funcional (fMRI). Nessa perspectiva,o artigo apresentará estudos dos três aspectos mais investigados em processamento sintático: a violação sintática; o processamento de sentenças comparado com lista de palavras; e a complexidade sintática (FRIEDERICI, 2004). A metodologia caracteriza-se por uma abordagem exploratória acerca do tema, alicerçada em pesquisa bibliográfica.
\end{abstract}

Palavras-chave:fMRI. Processamento sintático. Tarefas de compreensãoauditiva.

\begin{abstract}
This article aims at contributing to the understanding of the nature of language and the human brain by analyzing studies of syntactic processing. Studies involving syntactic processing are still a challenge for neuroscience. However, advanced techniques have been used to investigate how the brain stores and processes information, being able to construct a sentence. The present paper, studies which used comprehension tasks of syntactic structures together with fMRI are discussed. It addresses studies which investigated the three most studied aspects in syntactic processing: syntactic violation; processing sentences compared to the list of words; and syntactic complexity (FRIEDERICI, 2004). The methodology consists of an exploratory approach on the subject, based on a review.
\end{abstract}

Keywords: fMRI, syntactic processing, auditory comprehension tasks.

\section{Introdução}

Compreender o que está sendo dito não depende somente do significado das palavras, mas da relação estabelecida entre elas na frase. Tal habilidade de processar a linguagem tem despertado a curiosidade de vários pesquisadores nos últimos anos. Sendo assim, o processamento sintático começou a ser estudado recentemente pela neurociência com o objetivo de entender como funcionam os diferentes processos sintáticos no cérebro e quais as redes neurais responsáveis por eles.

A questão inicial é se podemos realmente separar processamento sintático de processamento semântico (FRIEDERICI, 2004). A gramática gerativa de Chomsky nos ajuda a pensar nessa questão. De acordo com a teoria linguística chomskiana, a linguagem é modular, desta maneira, sintaxe e semântica seriam módulos distintos da faculdade 
humana da linguagem. No entanto, apesar de o conhecimento sintático ser um sistema independente, ele resulta do conhecimento semântico-lexical. Simplificando, a sintaxe precisa das informações semânticas das palavras contidas no léxico para formar sentenças, já que no léxico estão as informações sobre categoria de palavra e estrutura de argumentos, imprescindíveis para a formação de frases.

Ullman (2001) apresenta uma visão recente de que as regras sintáticas são ligadas à memória procedural, localizada no córtex frontal e no gânglio basal, e de que o léxico está localizado no circuito temporal. Estudos que corroboram essa informação de Ullman são baseados no teste de processamento da flexão de verbos regulares (que representa a gramática, o sistema de regras) e da flexão de verbos irregulares (que são irregularidades armazenadas no léxico, representam, portanto, o léxico $)^{1}$. Segundo Friederici (2004), somente com esses testes de processamento de verbos, que são, obviamente, palavras, abre-se margem para pensar se a divisão "léxicoversus regras sintáticas" também acontece em nível de processamento sintático de sentenças e de processamento de processos semânticos que não estejam no nível da palavra (que não estariam, então, gravados no léxico).

Sendo assim, pesquisas recentes de fMRI que especificam diferentes áreas do cérebro para os processamentos léxico-semânticos e para os processamentos sintáticos durante a compreensão de sentenças sugerem que os processos semânticos ocorrem na região de BA 45/47 no giro frontal inferior e na região posterior do giro temporal médio, juntamente com a região posterior do giro temporal superior. Diferentemente dos processamentos sintáticos que envolvem áreas como o opérculo frontal e BA 44/45 no giro frontal inferior e a região anterior do giro temporal superior, além do gânglio basal e da região posterior do giro temporal superior.

Considerando que o processamento sintático pode ser investigado enquanto os participantes

\footnotetext{
${ }^{1}$ Em português, seria, por exemplo, a distinção entre comi, verbo com flexão regular de primeira pessoa do pretérito perfeito $-i$, e fiz, verbo ser irregular no pretérito perfeito.
}

desempenham uma tarefa de leitura (JUST, CARPENTER, KELLER, EDDY e THULBORN, 1996) ou uma tarefa de compreensão auditiva (SCHLOSSER et al., 1998), este trabalho tem o objetivo de discutir os três aspectos mais estudados em processamento sintático citados Friederici (2004) durante tarefas de compreensão auditiva. Na seção 2 analisamos estudos sobre violação sintática; processamento de sentenças comparado com lista de palavras; e complexidade sintática, verificando as áreas ativadas pelas análises de fMRI e o modo como o processamento sintático é estudado. Na seção 3 fazemos uma discussão sobre estes diferentes estudos e sobre o processamento exclusivamente sintático.

\section{0 processamento sintático da linguagem}

Grodzinsky e Friederici (2006) apresentam duas abordagens para o estudo do processamento sintático em neurociência. A primeira abordagem, o mapa formal sintático (FSM em inglês), estudada por Grodzinsky, busca a localização cerebral do conhecimento sintático humano e acredita que há uma relação entre os subcomponentes da teoria sintática e as suas localizações no cérebro. A hipótese que sustenta esta abordagem é justamente a de Chomsky, citada no início deste trabalho, que define operações (regras gramaticais) que dão estrutura para as sentenças. A segunda abordagem, o mapa do processamento da linguagem (LFM em inglês), estudada por Friederici, investiga os processos on-line envolvidos na análise da linguagem. A hipótese da LFM é a de que os mecanismos cerebrais lidam com diferentes fontes de conhecimento durante a análise on-line da sentença, fontes essas separadas em unidades neuronais. Segundo os autores, as duas abordagens são complementares e sugerem que subcomponentes do sistema de processamento da linguagem são distinguíveis e localizáveis no cérebro humano.

Os estudos que se propuseram a investigar unicamente a sintaxe encontraram dois estágios diferentes de processamento sintático: um processo 
inicial, de construção on-line da estrutura sintática; e um processo posterior, de checagem e reanálise, isto é, uma memória sintática para frases com lacunas ou com dependências sintáticas. Friederici (2004) constatou que o giro temporal superior esquerdo (STG), junto com o opérculo frontal, são responsáveis pelo processamento sintático on-line e que a parte anterior do giro frontal inferior (IFG) é responsável pela memória sintática de frases com grandes dependências e/ou lacunas ${ }^{2}$.

Os experimentos foram refinados desde os primeiros estudos com neuroimagem e, por isso, hoje o sistema linguístico pode ser neurologicamente localizado e decomposto em componentes e é possível investigar o processamento sintático. Sendo assim, segundo Friederici (2004), os aspectos mais pesquisados atualmente em processamento sintático são: a violação do processamento sintático, o processamento sintático de estruturas comparado com listas de palavras e a interação de complexidade sintática com memória. Nas subseções que seguem este trabalho, abordaremos como o processamento sintático pode ser investigado em cada um desses aspectos e quais regiões do cérebro são ativadas em cada um.

\subsection{A violação do processamento sintático}

As violações sintáticas provocam ativação no opérculo frontal e na ínsula, ambos no hemisfério esquerdo, enquanto a identificação de erros sintáticos ativa a área de Broca. Friedericiet al. (2003) conduziram um estudo empírico com 15 falantes adultos do alemão sobre violação sintática e violação semântica para verificar a interdependência desses processos e a área ativada por ambos.

Para posterior análise dos processos de violação na compreensão de sentenças, os 15 indivíduos alemães, todos destros, foram expostos a estímulos auditivos. Os participantes ouviam uma

\footnotetext{
${ }^{2}$ Exemplos em português para estes dois processos sintáticos seriam: para o primeiro, uma frase na ordem direta, em que a memória sintática não precisa ser acessada, como João ama Maria; para o segundo, uma sentença com uma frase encaixada, em que a memória sintática é necessária para, por exemplo, ligar o sujeito ao objeto, como $A$ menina que o João gosta mais do que gosta da sua mãe é a Maria.
}

sentença em alemão e deviam apertar o botão de 'sim' se julgassem que a frase estava correta e o botão de 'não' se julgassem a frase como incorreta. As condições da tarefa eram quatro: (1) sentenças que são sintática e semanticamente corretas; (2) sentenças com violação sintática somente; (3) sentenças com violação semântica somente; (4) sentenças "filler", de preenchimento, sentenças preposicionais completas e corretas, colocadas para evitar que os participantes descobrissem qual era o objetivo da tarefa.

As frases foram randomizadas aleatoriamente para cada participante, com o mesmo número de sentenças de cada condição para todos os participantes. As frases corretas eram frases em alemão, de verbos transitivos, na forma passiva, no tempo imperfeito: "Das Hemdwurdegebügelt (The shirtwasironed)". Nas frases da condição (2), de violação sintática, o verbo no particípio imediatamente seguia a preposição, o que gera uma ordem inexistente em alemão: "Die Blusewurdeamgebügelt (The blousewasonironed)". Nas frases da condição (3), de violação semântica, o significado do verbo no particípio não era congruente com o significado do resto da sentença: "Das Gewitterwurdegebügelt (The thunderstormwasironed)". As frases da condição (4), condição de preenchimento, continham um sintagma preposicional bem formado, assim como uma construção de particípio bem formada também: "Der Rock wurdeamFreitaggebügelt (The skirtwason Friday ironed)". As frases dessa condição de preenchimento "fillercondition" não foram incluídas na análise final dos dados de fMRI, mas elas foram incluídas no experimento para que o participante não pudesse prever a violação sintática baseado somente na presença equivocada da preposição. As sentenças para exemplificar cada condição assim como as suas traduções em inglês foram retiradas do artigo de Friedericiet al. (2003), as traduções mantém a ordem da frase em alemão.

Com os resultados deste experimento, chegase à conclusão de que há similaridades e diferenças interessantes entre processamentos de violação sintática e semântica. As duas violações ativam 
porções da região temporal superior mais do que sentenças corretas. Os resultados corroboram estudos anteriores que postulam que ambos os processamentos, sintáticos e semânticos, ocorrem em uma rede temporal frontal, no entanto, cada processamento possui áreas específicas distintas (Tabela 1) (INDEFREY et al., 2001 e MORO et al., 2001).

\subsection{O processamento sintático de estruturas comparado com listas de palavras}

O processamento sintático de estruturas comparado com o processamento de listas de palavras mostra que essas distintas tarefas ativam áreas diferentes. Nas condições em que a sintaxe está presente há ativação da região anterior do giro temporal superior (STG), fato não observado para a tarefa de processamento de listas de palavras.

O processamento sintático de estruturas e listas de palavras é abarcado no estudo conduzido por Friederici, Meyer e Cramon (2000). A pesquisa teve como objetivo identificar as regiões cerebrais específicas no processamento de informações sintáticas e semânticas durante a compreensão de discursos. Os estímulos variavam sistematicamente entre a ausência e a presença de informações estruturais e de palavras com conteúdo. Para tanto, 18 indivíduos destros foram expostos a quatro tipos distintos de estímulos auditivos. Sendo eles: (1) sentenças que são sintática e semanticamente corretas; (2) discursos sintaticamente corretos em que as palavras de conteúdo são substituídas por pseudopalavras; (3) listas de palavras reais sem estrutura sintática, mas com informação semântica; (4) listas de pseudopalavras sem informações sintáticas e semânticas.

As condições (1) e (2) consistem na apresentação de sentenças, enquanto as condições (3) e (4) consistem na apresentação de listas de palavras. As listas de palavras são construídas da seguinte forma: o número de palavras corresponde ao número de palavras nas sentenças sendo que a primeira palavra de cada estímulo consiste em um artigo, de modo a manter a mesma categoria. As palavras seguintes na lista de palavras reais são adjetivos, advérbios e substantivos. Na condição de listas de pseudopalavras, as palavras seguintes a primeira palavra (artigo) são palavras que não têm significado, mas que respeitam as regras fonotáticas. Os estímulos são apresentados randomicamente para equilibrar os efeitos de sequência. Além disso, para manter a atenção dos sujeitos enquanto escutam ao áudio, eles são solicitados pelos pesquisadores a executar uma tarefa de julgamento após cada estímulo. A fim de manter a tarefa constante para as diferentes condições, os sujeitos são solicitados a indicar se o estímulo ouvido contém uma estrutura sintática ou palavras de conteúdo.

Os resultados de fMRI indicam que o padrão de ativação varia em função do tipo específico do estímulo apresentado aos participantes. Os pesquisadores constataram que o processamento sintático está relacionado com o aumento da ativação na região posterior do giro temporal superior esquerdo e na região anterior do giro temporal superior bilateral. Tal aumento na ativação foi observado nas condições que envolveram sentenças e discursos se comparado às condições que somente listas de palavras foram apresentadas. No entanto, o efeito semântico não foi observado nas condições investigadas.

\subsection{A interação de complexidade sintática com memória}

Ao comparar frases mais complexas com frases menos complexas, os pesquisadores conseguiram focar somente nos processos sintáticos. Através da apresentação de estruturas sintáticas mais complexas, pôde-se observar um aumento na ativação de regiões como a área de Broca e Wernicke. Segundo os pesquisadores, tal aumento de ativação nessas áreas deve-se ao fato de que quanto mais complexa for uma estrutura sintática, maior será o número de recursos cognitivos desprendidos para realizar uma tarefa. 
Além disso, estudos sugerem que as regiões superior e anterior do giro frontal inferior (BA44/45) são ativadas em processos de compreensão que envolvemestruturas não canônicas, ou seja, a demanda de memória de trabalho é maior para estruturas não canônicas se comparadas com as canônicas (FIEBACH, SCHLEWESKY \& FRIEDERICI, 2004). Ainda, Roederet al. (2002) concluíram que a complexidade sintática favorece 0 aumento de ativação no giro frontal inferior (IFG) e no giro temporal superior e médio.

O processamento sintático da interação de complexidade sintática com a memória é discutido nos artigos de Segaertet al. (2012, 2013). Os pesquisadores realizaram estudos em que os participantes teriam de compreender ou produzir frases na voz ativa ou na passiva, sendo a segunda condição considerada de alta complexidade. Foram realizadas com os participantes tarefas de produção e compreensão de sentenças com o objetivo de verificar o efeito depriming sintático.

Priming é o processamento automático e préconsciente de informações. É um fenômeno que ocorre quando o processamento de uma informação armazenada na memória é facilitado pela apresentação de um estímulo prévio - o prime (BLANK, 2008). Durante a realização de tarefas de priming, estímulos (primes) em forma de imagens, palavras, sons, sentenças ou objetos são utilizados para sensibilizar o sujeito a uma apresentação posterior de outro estímulo (alvo). A percepção desses estímulos prime, nessas tarefas, é automática e ocorre de forma inconsciente. Vários tipos de experimentos podem ser realizados dentro dos estudos de priming, de acordo com os propósitos a serem investigados. Assim, podem-se criar experimentos nos quais prime e alvo são apresentados com ou sem semelhanças semânticas, grafêmicas, fonético-fonológicas, entre outras. A evidência empírica do efeito de priming é obtida na execução de uma tarefa cuja a facilitação seja demonstrada, abrangendo o processamento da percepção pré-consciente e da memória implícita.
Desse modo, nas tarefas de compreensão e produção de frases de Segaertet al (2013), o prime consiste na apresentação de um verbo na forma infinitiva na cor cinza, na tarefa de compreensão, e na cor verde, na tarefa de produção. O verbo, em cinza ou em verde, é acompanhado de uma foto de um casal representando a ação desses verbos, juntamente com o áudio descrevendo a ação, na tarefa de compreensão, ou juntamente com a solicitação de produção de uma sentença utilizando o verbo apresentado para descrever a ação vista na foto. A compreensão e produção podem ser tanto na voz ativa como na voz passiva. Esses dois primeiros estímulos de compreensão (cinza) e produção (verde) representam o prime e antecedem o verbo e a foto alvo, ou seja, depois do primeiro par verbo e foto, um outro verbo seguido de uma outra foto são apresentados. Se o estímulo for de compreensão, o participante escuta ao áudio da segunda foto e deve pressionar o botão somente se o áudio não corresponder à foto. Se for de produção, o participante deve formular uma sentença de modo que mencione o ator que estiver em verde primeiro na frase, podendo o ator em verde estar executando a ação (voz ativa) ou sofrendo a ação (voz passiva). A apresentação dos estímulos se dá de forma randomizada, ou seja, o prime pode ser tanto de compreensão como de produção seguido de um alvo que pode ser tanto de compreensão quanto de produção. Além disso, a tarefa possui condições como: 1) sem repetição de verbo; 2) com repetição sintática, mas sem repetição de verbo; 3) com repetição de verbo, mas sem repetição sintática; e 4) com repetição verbal e sintática. Sendo que nenhuma condição é repetida duas vezes seguidas e as condições são apresentadas de forma randomizadas. Ainda, o tempo de resposta é medido em milissegundos e a acurácia (o escore de acerto), em porcentagem.

Através dessa tarefa de compreensão e produção de sentenças, estes pesquisadores concluíram que as bases neurais do processamento sintático para compreensão e produção de fala são compartilhadas. A pesquisa apontou as mesmas 
regiões neurais mencionadas no estudo de Friederici 2004, o giro frontal inferior (IFG) e o giro temporal medial (MTG). Desse modo, segundo o estudo, é no giro temporal medial que está codificado e consolidado todo o nosso conhecimento linguístico, mas é o giro frontal inferior que combina o conhecimento linguístico em diferentes maneiras e faz a montagem das estruturas com a contribuição do contexto. Os resultados encontrados nesta pesquisa estabelecem uma relação entre estruturas sintáticas mais complexas e o aumento de ativação no giro frontal inferior mediante à demanda do processamento sintático, corroborando os achados citados na revisão de Friederici (2004).

\section{Análises e Conclusão}

A Tabela 1 mostra as áreas ativadas para cada tipo de processamento sintático estudado pelos artigos apresentados neste artigo. A partir dela, podemos extrair conclusões acerca dos diferentes estudos de processamento sintático, ainda recentes em neurociência.

A leitura dessa tabela nos leva a uma importante conclusão. Podemos ver, a partir do estudo (i) sobre violações, que o giro temporal superior posterior esquerdo e o giro temporal superior posterior direito estão envolvidos no processamento da informação de restrição selecional do verbo, isto é, argumentos que o verbo exige/seleciona. Deste modo, o giro temporal superior posterior parece ser a região que suporta a integração da informação lexical com a informação sintática. A sintaxe possivelmente "pega" as informações semânticas (restrição semântica, como [+animado]) e sintáticas (tipos de argumentos, como sintagma nominal, sentença) das palavras e projeta em uma estrutura sintática, em uma sentença. Quando ocorre violação na seleção argumental do verbo, o giro temporal superior posterior é ativado, pois não consegue projetar devidamente as informações em uma sentença.

Assim, o processamento sintático parece realmente precisar de informações léxico-semânticas para formar sentenças, no entanto este

Tabela 1: Aspectos sintáticos pesquisados e as áreas ativadas

\begin{tabular}{|c|c|c|c|}
\hline ESTUDOS fMRI & ASPECTOS & ÁREAS ATIVADAS & TIPOS ESTÍMULOS \\
\hline $\begin{array}{l}\text { FRIEDERIC, et } \\
\text { al., } 2003 .\end{array}$ & $\begin{array}{l}\text { (i) } \\
\text { Violação do } \\
\text { processamento } \\
\text { sintático }\end{array}$ & $\begin{array}{l}\text { Violação sintática: } \\
\text { - giro temporal superior posterior esquerdo } \\
\text { - giro temporal médio superior esquerdo } \\
\text { - giro temporal superior anterior esquerdo } \\
\text { - giro temporal superior posterior direito } \\
\text { - opérculo frontal posterior esquerdo } \\
\text { - giro supramarginal esquerdo } \\
\text { - gânglio basal esquerda } \\
\text { Violação semântica: } \\
\text { - giro temporal superior esquerdo } \\
\text { - giro temporal superior posterior esquerdo } \\
\text { - giro temporal médio superior direito } \\
\text { - córtex premotor inferior direito } \\
\text { - insula anterior esquerda } \\
\text { - insula anterior direita }\end{array}$ & $\begin{array}{l}\text { (1) sentenças que são } \\
\text { sintática e } \\
\text { semanticamente } \\
\text { corretas; } \\
\text { (2) sentenças com } \\
\text { violação sintática } \\
\text { somente; } \\
\text { (3) sentenças com } \\
\text { violação semântica } \\
\text { somente; } \\
\text { (4) sentenças "filler" }\end{array}$ \\
\hline $\begin{array}{l}\text { FRIEDERICI, et } \\
\text { al., } 2000 .\end{array}$ & $\begin{array}{l}\text { (ii) } \\
\text { Processamento } \\
\text { sintático de } \\
\text { sentença x } \\
\text { processamento de } \\
\text { lista de palavras }\end{array}$ & $\begin{array}{l}\text { - giro temporal superior esquerdo } \\
\text { - giro temporal superior anterior bilateral } \\
\text { - córtex auditivo anterior } \\
\text { - opérculo frontal esquerdo }\end{array}$ & $\begin{array}{l}\text { (1) sentençascorretas; } \\
\text { (2) sintaticamente } \\
\text { corretos com uso de } \\
\text { pseudopalavras; } \\
\text { (3) listas sem estrutura } \\
\text { sintática, mas com } \\
\text { informação semântica; } \\
\text { (4) listas de } \\
\text { pseudopalavras. }\end{array}$ \\
\hline $\begin{array}{l}\text { SEGAERT, K., } \\
\text { et al., } 2012 .\end{array}$ & $\begin{array}{l}\text { (iii) } \\
\text { A interação de } \\
\text { complexidade } \\
\text { sintática com } \\
\text { memória }\end{array}$ & $\begin{array}{l}\text { - giro temporal médio esquerdo } \\
\text { - giro frontal inferior } \\
\text { - áreamotorasuplementar bilateral }\end{array}$ & $\begin{array}{l}\text { 1) sem repetição de } \\
\text { verbo; } \\
\text { 2) com repetição } \\
\text { sintática, mas sem } \\
\text { repetição de verbo; } \\
\text { 3) com repetição de } \\
\text { verbo, mas sem } \\
\text { repetição sintática; } \\
\text { 4) com repetição verbal } \\
\text { e sintática. }\end{array}$ \\
\hline
\end{tabular}


processamento pode ser também visto exclusivamente. Um bom exemplo de investigação do processamento sintático é o estudo (iii) sobre complexidade sintática, que compara sentenças menos e mais complexas (ativas e passivas respectivamente), conseguindo, então extrair o processamento sintático "puro". Nesse estudo o giro temporal superior posterior não parece ser ativado, as áreas ativadas são o giro temporal médio esquerdo, o giro frontal inferior. Segundo a pesquisa, enquanto o giro temporal medial auxilia na recuperação de informações léxico-sintáticas da memória, o giro frontal inferior auxilia na unificação dessas informações.

Neste trabalho, procuramos delinear os pontos importantes dos estudos sobre processamento sintático. No entanto, o importante é não esquecer o objetivo de todos os estudos que se pretendem "estudos linguísticos": o que nós sabemos quando sabemos uma língua? São as respostas dessa pergunta que devem ser investigadas no cérebro humano e, com certeza, "a estrutura das frases dessa língua" é uma das respostas.

\section{Referências}

BLANK, Cintia, A. A transferência grafo-fônicofonológica L2 (francês) - L3 (inglês): um estudo conexionista. 2008. 148p. Pelotas, RS. Dissertação de Mestrado. UniversidadeCatólica de Pelotas UCPel.

FIEBACH, Christian. J., SCHLESEWSKY, Matthias; BORNKESSEL, Ina.D., von CRAMON, D. Yves, FRIEDERICI, Angela. D. Distinct neural correlates of legal and illegal word order variations in German: How can fMRI inform cognitive models of sentence processing? In On-line Study of Sentence Comprehension - Eyetracking, ERPs and Beyond, p. 357-370, 2004.

FRIEDERICI, Angela. D. The Neural Basis of Syntactic Processes. In: The Cognitive Neurosciences III, MIT. p. 789- 801, 2004.

FRIEDERICI, Angela. D.; MEYER, Martin.; von CRAMON, D. Yves. Auditory language comprehension: An event-related fMRI study on the processing of syntactic and lexical information. Brain Language, v. 74, n. 2, p. 289-300, dezembro, 2000.

FRIEDERIC, Angela D.; RÜSCHMEYER, Shirley A.; HAHNE, Anja; FIEBACH, Christian J. The Role of Left Inferior Frontal and Superior Temporal Cortex in Sentence Comprehension: Localizing Syntatic and
Semantic Processes. Cerebral Cortex, v. 13, n.2, p. $170-177,2003$.

GRODZINSKY, Yosef; FRIEDERICI, Angela. D. Neuroimaging of syntax and syntactic processing.Current Opinion in Neurobiology, v. 16, p. 240-246, 2006.

INDEFREY, Peter, HAGOORT, Peter, HERZOG, Hans, SEITZ, Rudiger, J., BROWN, Colin, L. Syntactic processing in left prefrontal cortex is independent of lexical meaning. Neurolmage, v. 14, n. 3, p. 546-555, 2001.

JUST, Marcel A.; CARPENTER, Patricia A.; KELLER, Timothy A.; EDDY, William F.; THULBORN, Keith R. Brain activation modulated by sentence comprehension. Science, v.274, p.114-116, 1996.

MORO, Andrea; TETTAMANTI, Marco; PERANI, Daniela; DONATI, Caterina; CAPPA, Stefano F; FAZIO, Ferruccio. Syntax and the brain: Disentangling grammar by selective anomalies. Neurolmage, v. 13, n. 1, p. 110-118, 2001.

ROEDER, Brigitte; STOCK, Oliver; NEVILLE, Helen; BIEN, Siegfried; ROESLER, Frank. Brain activation modulated by the comprehension of normal and pseudo-word sentences of different processing demands: A functional magnetic resonance imaging study. Neurolmage, v. 15, n. 4, 1003-1014, 2002.

SCHLOSSER, Michael. J.; AOYAGI, Nobuhisa; FULLBRIGHT, Robert K.; GORE, John. C.; McCARTHY, Gregory. Functional MRI studies of auditory comprehension. Human Brain Mapping, v. 6, n. 1, p.1-13, 1998.

SEGAERT, Katrien; MENENTI, Laura; WEBER, Kirsten; PETERSSON, Karl M.; HAGOORT, Peter. Shared syntax in language production and language comprehension - An fMRI study. Cerebral Cortex, v. 22, p. 1662-1670, 2012.

SEGAERT, Katrien; KEMPEN, Gerard; PETERSSON, Karl M.; HAGOORT, Peter. Syntactic priming and the lexical boost effect during sentence production and sentence comprehension: An fMRI study. Brain \& Language, v. 124, n. 2, p. 174-183, 2013.

ULLMAN, Michael. The declarative/Procedural model of lexical and grammar.Journal of Psycholinguistic Research, v. 30, n. 1, p. 37-69, 2001. 